\title{
Tromboprofilaxia em Pacientes Neurocirúrgicos: Uma Revisão Integrativa da
}

\section{Literatura}

\section{Thromboprophylaxis in Neurosurgical Patients: An Integrative Literature Review}

Katerine Costa Bambeko Rodrigues ${ }^{1}$, Kaiomakx Renato Assunção Ribeiro ${ }^{2}$, Roselaine Prota Baudson da Silva ${ }^{3}$, Mikaelle Roane Modesto Pereira ${ }^{4}$, Oriana Katia Fracalossi ${ }^{5}$, Glayson Carlos Miranda Verner ${ }^{6}$, Jacqueline Ramos de Andrade Antunes Gomes ${ }^{7}$.

1- Enfermeiro. Especialista em Centro Cirúrgico. Escola Superior de Ciências da Saúde do Distrito Federal- ESCS-FEPECS/SES/DF - Brasília (DF), Brasil. E-mail: katerinecbrodrigues@gmail.com.

2- Enfermeiro. Especialista em Unidade de Terapia Intensiva, Cardiologia e Hemodinâmica. Instituto Hospital de Base do Distrito Federal. Brasília (DF), Brasil. E-mail: kaiomakxribeiro@hotmail.com.

3- Enfermeira. Especialista em Centro Cirúrgico. Escola Superior de Ciências da Saúde do Distrito Federal- ESCS-FEPECS/SES/DF - Brasília (DF), Brasil. E-mail: roseprota@gmail.com.

4-Enfermeira. Especialista em Centro Cirúrgico. Escola Superior de Ciências da Saúde do Distrito Federal- ESCS-FEPECS/SES/DF - Brasília (DF), Brasil. E-mail: mikaelle.roane@gmail.com.

5- Enfermeira. Especialista em Centro Cirúrgico. Escola Superior de Ciências da Saúde do Distrito Federal- ESCS-FEPECS/SES/DF - Brasília (DF), Brasil. E-mail: ori.fracalossi@gmail.com.

6- Médico Anestesiologista. Chefe da Unidade de Anestesiologia e Medicina Perioperatória do Hospital Regional do Gama/DF- Brasília (DF), Brasil. Email: glayson.verner@ hotmail.com.

7- Enfermeira. Doutora em Ciências da Saúde. Coordenadora da Residência de Enfermagem em Centro Cirúrgico em Rede. Escola Superior de Ciências da Saúde do Distrito FederalESCS-FEPECS/SES/DF - Brasília (DF), Brasil. E-mail: jacquelinecchbdf@ gmail.com.

\section{Resumo}

Objetivo: Identificar na literatura o tipo de método mais utilizado para tromboprofilaxia em neurocirurgia. Métodos: Trata-se de uma revisão integrativa da literatura. Para elaboração desta pesquisa foram utilizados artigos científicos, disponibilizados nas bases de dados PUBMED/MEDLINE, LILACS e SciELO, publicados entre 2010 e 2019. Resultados: Para a discussão dos resultados, foram selecionados 08 artigos utilizando os critérios de inclusão préestabelecidos. Na maioria dos estudos, se associam mais de um método tromboprofilático, seja o uso de meias compressivas, compressão pneumática intermitente, heparina de baixo peso molecular, heparina não fracionada ou novos anticoagulantes orais, no entanto, alguns métodos farmacológicos ainda não são totalmente seguros para o uso, pois, como enfatizado nos estudos aqui mostrados, os casos de profilaxia farmacológica precisam ser avaliados individualemente. Conclusão: Mais investigações são necessárias para avaliar de fato qual o método mais seguro para se utilizar em neurocirurgia.

Palavras-chave: Tromboprofilaxia, Farmacologia, Neurocirurgia 


\begin{abstract}
Objective: To identify in the literature the type of method most used for thromboprophylaxis in neurosurgery. Methods: For the elaboration of this research were used scientific articles, available in the databases Pubmed/MEDLINE, LILACS and SciELO, published between 2010 and 2019. Results: For the discussion of the results, 08 articles were selected using the inclusion criteria pre-established. In the majority of studies associate more than one tromboprofilático method, is the use of compressive stockings, Intermittent pneumatic compression, low molecular weight heparin, unfractionated heparin or oral anticoagulants, however the pharmacological methods are not yet totally safe to use, because, as emphasized in the speeches of the studies shown here, the cases of pharmacological prophylaxis must be evaluated individually. Conclusion: More investigations are necessary to evaluate which is the safest method to be used in neurosurgery.
\end{abstract}

Keywords: Thromboprophylaxis, Pharmacological, Neurosurgical

\title{
INTRODUÇÃO
}

As trombofilias podem ser tanto hereditárias como também adquiridas e nos casos de hipercoagulabilidade secundária há diversas patologias adquiridas que predispõem os pacientes a um evento trombótico (Câncer, Insuficiência Cardíaca (IC), Infarto Agudo do Miocárdio (IAM), Obesidade, Imobilização, Tromboembolismo Venoso prévio, Anticoncepcionais, Idade, Cirurgias de Grande Porte, etc.) adquiridas que predispõem os pacientes a um evento trombótico. ${ }^{(1-3)}$

A Trombose Venosa Profunda (TVP) é uma complicação desse estado de hipercoagulabilidade, culminando na formação de trombos, que podem ou não ocluir os vasos do sistema venoso. Virchow no século XIX descreveu que esse mecanismo dependia de 3 fatores: a hipercoagulabilidade, estase sanguínea e lesão endotelial, formando assim a conhecida tríade de Virchow. No caso da Embolia Pulmonar (EP) este trombo que se formou no sistema venoso profundo se desprende, ganha a circulação cardíaca até atingir a artéria pulmonar, gerando uma oclusão deste vaso. ${ }^{(1-3)}$

O Tromboembolismo Venoso (TEV) é uma das causas mais comuns de morbimortalidade de paciente politraumatizados, e a incidência de trombose pós-operatória é cerca de $20 \%$ a $25 \%$ e $2 \%$ dos pacientes evoluem para embolia pulmonar. A incidência geral é de 5/10.000 pacientes, sendo quatro vezes maior a mortalidade se o paciente não tiver 
tratamento em curso. Em torno de 100.000 pacientes vem a óbito por TEP nos Estados Unidos por ano. Segundo o House of Commons Health Select Committe, 25000 pessoas morrem no Reino Unido de TEV, a cada ano, gerando grande impacto financeiro ao sistema de saúde. ${ }^{(1,3-}$ 4)

A tromboprofilaxia seja mecânica ou farmacológica é a maneira mais eficaz de se reduzir a morbimortalidade do TVP e da TEP em pacientes no pós-operatório, principalmente pela imobilidade no leito, após o procedimento. O uso da profilaxia mecânica é justificado naqueles pacientes com alto risco de sangramento no pós-operatório, sendo de grande relevância seu uso em paciente neurocirúrgicos, associados a outros métodos farmacológicos ${ }^{(3-}$ 5)

Cerca de $50 \%$ dos pacientes neurocirúrgicos podem ter um evento trombótico, no entanto não há um consenso em relação à tromboprofilaxia farmacológica, isto se deve ao fato de que estes pacientes tem uma alta mortalidade e grandes chances de sangramento, podendo a terapêutica gerar danos permanentes. A utilização de meias compressivas e a Compressão Pneumática Intermitente, têm sido utilizadas em grande escala para os pacientes neurocirúrgicos. Já os métodos farmacológicos são descontinuados em torno de 5 a 7 dias antes do procedimento cirúrgico para retorno da coagulação. ${ }^{(5,6)}$

A escolha do método farmacológico depende do tipo de procedimento neurocirúrgico ao qual o paciente vai ser submetido, do curso da cirurgia e a análise do risco-benefício. Os riscos do TEV/TEP variam se o paciente possui alguma neoplasia cerebral, se têm alguma lesão traumática cerebral, hemorragia subaracnóidea, hemorragia intracerebral, ou lesão aguda da medula espinal. Deve-se levar em consideração que os pacientes neurocirúrgicos e neurocríticos podem ter outros fatores de risco e doenças prévias como por exemplo válvula cardíaca, fibrilação atrial com tromboembolismo, história de trombose venosa profunda, trombofilia, ou Stent da artéria coronária. ${ }^{(5,6)}$ 
Quanto ao Novos Anticoagulantes Orais, por exemplo rivoroxaban, apixabano, etexilato, edoxaban, tem o uso mais seguro que a warfarina por terem uma farmacocinética mais estável.

Os novos anticoagulantes são cada vez mais prescritos e algumas evidências de que asseguram menor risco de hemorragia intracraniana, no entanto, por falta de exames que avaliem prontamente a anticoagulação e ausência de reversores prontamente disponíveis como também o alto custo, o uso sem cautela predispõe o paciente a riscos. ${ }^{(7,8)}$

Por se tratar de um assunto global de grande relevância, o tema deste artigo foi escolhido com base na vivência de 4 meses em um Ambulatório de Anestesia de um Hospital Público do Distrito Federal, onde se realizavam diversas estratificações para avaliar o risco de TVP e TEP em pacientes que iriam se submeter a procedimentos cirúrgicos. O objetivo geral deste artigo é identificar na literatura o tipo de método mais utilizado para tromboprofilaxia em neurocirurgia.

\section{METODOLOGIA}

Trata-se de uma revisão integrativa da literatura (RIL) com objetivo de analisar o que se tem construído em pesquisas anteriores sobre um determinado tema. Sendo a questão norteadora: Qual a tromboprofilaxia indicada para os pacientes neurocirúrgicos?

Para elaboração desta pesquisa foram utilizados artigos científicos, disponibilizados nas bases de dados National Libraly of Medicine (MEDLINE) via PUBED, Literatura Latinoamericana e do Caribe em Ciências da Saúde (LILACS) e na Scientific Electronic Library Online (SciELO) que contivessem nos títulos ou resumos dos Descritores em Ciências da Saúde (DeCS): thromboprophylaxis pharmacological, neurosurgical, tromboembolism. Para ampliar a busca da literatura o indicador boleano AND foi utilizado entre os descritores. 
Como critérios de inclusão foram incluídos textos que abordavam a tromboprofilaxia em neurocirurgias, publicados entre 2010 e 2019 nos idiomas inglês, português e espanhol e que se encontravam disponíveis na íntegra.

Foram excluídas teses, dissertações, editoriais, artigos que não tratavam sobre o assunto. Artigos disponíveis em 2 ou mais bases de dados foram considerados apenas uma vez. Foram obtidos 17 artigos, o quais foram submetidos a leitura exploratória do título e resumo, e em seguida foi realizada a leitura completa dos artigos.

\section{RESULTADOS}

Foram encontrados incialmente 629 artigos. Após aplicação dos critérios de inclusão e exclusão resultaram em 25 artigos. Após a leitura completa dos estudos, considerou-se para essa revisão um total de 8 estudos, os quais estão dispostos no Quadro 1 com o título, ano de publicação e síntese dos resultados.

Quadro 1: Resultados encontrados nos estudos incluídos nesta revisão.

\begin{tabular}{|c|c|}
\hline Autores, Ano & Resultados dos estudos \\
\hline $\begin{array}{l}\text { Artigo 1: } \\
\text { Alonso et al, } 2015^{5}\end{array}$ & $\begin{array}{l}\text { Dos } 73 \text { hospitais incluídos no Catálogo Nacional de Hospitais, uma resposta válida ao } \\
\text { questionário on-line foi recebida por } 41 \text { anestesiologistas de } 37 \text { locais (taxa de resposta } \\
50,7 \% \text { ). Somente uma resposta por site foi usada. Um protocolo específico estava } \\
\text { disponível em } 27 \% \text { desses centros. A tromboprofilaxia mecânica é utilizada no intra ou } \\
\text { pós-operatório em } 80 \% \text { e o tratamento farmacológico é utilizado por } 75 \% \text { dos } \\
\text { entrevistados. A enoxaparina foi a heparina mais frequente em pacientes } \\
\text { com craniotomia ( } 78 \% \text { ). As craniotomias foram realizadas mantendo o tratamento com } \\
\text { ácido acetilsalicílico em pacientes com stents coronários e } \\
\text { duplo tratamento antiplaquetário em metade dos centros. }\end{array}$ \\
\hline $\begin{array}{l}\text { Artigo 2: } \\
\text { Niemi, Armstrong, } \\
2010^{6}\end{array}$ & $\begin{array}{l}\text { A tromboprofilaxia farmacológica ou terapia de ponte deve ser adaptada de acordo com } \\
\text { os riscos individuais e o tipo de neurocirurgia. O risco de sangramento é provavelmente } \\
\text { minimizado por permitindo que a capacidade de coagulação se normalize no pré- } \\
\text { operatório e usando doses reduzidas de heparina de baixo peso molecular (HBPM) com } \\
\text { início relativamente tardio após neurocirurgia. A tromboprofilaxia mecânica pode ser } \\
\text { utilizada na maioria neurocirurgia e combinado com HBPM como o risco de } \\
\text { tromboembolismo é alto. }\end{array}$ \\
\hline $\begin{array}{l}\text { Artigo 3: } \\
\text { Agarwal et al, } 2019 \\
9\end{array}$ & $\begin{array}{l}\text { Um total de } 70 \text { TEVs }(0,6 \% \text { no total) foi documentado ( } 28 \text { tromboses venosa profunda, } \\
42 \text { embolias pulmonar). As maiores taxas de TEV foram associadas à deformidade } \\
(6,7 \%) \text {; cirurgia cerebrovascular aberta }(6,5 \%) \text {; hematoma subdural, intracerebral e } \\
\text { epidural }(3,2 \%) \text {; trauma raquimedular }(2,4 \%) \text {; e craniotomia para casos de tumor } \\
(1,6 \%) \text {. Sete casos de trombose venosa profunda progrediram para embolias } \\
\text { pulmonares, e } 66 \text { de } 70 \text { TEVs ocorreram durante o Tromboprofilaxia farmacológico. } 54 \\
\text { complicações hemorrágicas ocorreram após POD2 após o início da } \\
\text { tromboprofilaxia. Essas complicações hemorrágicas consistiram em novas hemorragias } \\
\text { observadas clínica ou radiograficamente. E } 28 \text { das } 54 \text { complicações hemorrágicas } \\
\text { tardias necessitaram de intervenção cirúrgica, com } 1 \text { morte. Quarenta e cinco pacientes } \\
\text { estavam em anticoagulação quando ocorreu o evento hemorrágico inicial. }\end{array}$ \\
\hline
\end{tabular}


\begin{tabular}{lllll}
\hline Artigo 4: & Drogas anti-hemostáticas e anticoagulantes demonstraram estar associadas ao & ad \\
Robba et al, $2017^{7}$ & sangramento perioperatório. Por outro lado, um risco aumentado
\end{tabular} de tromboembolismo venoso e estado hipercoagulativo após neurocirurgia eletiva e de emergência, em particular após cirurgia de tumor cerebral, foi descrito em vários pacientes. Para equilibrar o risco entre trombose e sangramento, é importante estar familiarizado com as alterações perioperatórias da coagulação e com as recentes diretrizes de manejo para pacientes anticoagulados submetidos a procedimentos neurocirúrgicos, em particular para aqueles que tomam novos anticoagulantes diretos. Foram considerados os ensaios clínicos e a literatura atuais sobre segurança e eficácia da profilaxia para trombose venosa profunda na população neurocirúrgica. Estes foram principalmente ensaios em pacientes eletivos cirúrgicos e de terapia intensiva com sangramento intracraniano de baixo grau ou traumas múltiplos com lesão cerebral traumática grave (TCE) associada.

Artigo 5:
Croci et al, $2017^{8}$

Artigo 6:
Chibbaro et al,
2018

A taxa de sangramento perioperatório foi de 13,3\% ( $\mathrm{n}=4$ de 30). O sangramento pósoperatório levou à morte em 2 pacientes. O tempo médio de descontinuação foi significativamente menor nos pacientes que apresentaram um evento hemorrágico em comparação àqueles sem um evento hemorrágico (1,5 dias [intervalo de 0 a 3 dias] vs. 11 dias [intervalo de 0 a 120 dias]). A taxa de eventos tromboembólicos perioperatórios foi de 3,3\% $(n=1)$ e a mortalidade geral foi de $13,3 \%(n=4)$.

No momento da análise, os dados obtidos dos pacientes incluídos neste estudo antes de al, 2014 (protocolo A, 3169 pacientes) foram comparados com os obtidos após a introdução do Compressão Pneumática Intermitente (CPI) (protocolo B, 3818 pacientes). Entre os pacientes designados para o protocolo A, 73 (2,3\%) desenvolveram trombose venosa profunda (TVP) e $28(0,9 \%)$ desenvolveram embolia pulmonar (EP), 9 dos quais foram fatais $(0,3 \%)$. Entre os pacientes designados para o protocolo $\mathrm{B}, 32$ desenvolveram TVP $(0,8 \%)$ e $7(0,18 \%)$ desenvolveram EP, com 2 eventualmente resultando na morte do paciente. Uma análise post-hoc confirmou que o uso de HBPM no pré-operatório não estava associado a um risco estatisticamente significativo de maior sangramento no pós-operatório.

\begin{tabular}{|c|c|c|}
\hline $\begin{array}{l}\text { Artigo 7: } \\
\text { Bekelis, } \\
\text { Labropoulos, } \\
2017^{12}\end{array}$ & Coy, & $\begin{array}{l}\text { Durante o período do estudo, } 94.947 \text { pacientes foram submetidos a procedimentos } \\
\text { neurocirúrgicos e preencheram os critérios de inclusão. Destes, } 1358(1,0 \%) \\
\text { desenvolveram TEV nos } 30 \text { dias pós-operatórios. A regressão logística multivariável } \\
\text { demonstrou uma associação de maior duração operatória com maior incidência de TEV } \\
\text { em } 30 \text { dias (odds ratio [OR], 1,22; intervalo de confiança de } 95 \% \text { [IC], 1,19- } \\
\text { 1,25). Comparados com procedimentos de duração moderada (terceiro quintil, percentil } \\
\text { 40-60), os pacientes submetidos aos procedimentos mais longos (> percentil 80) } \\
\text { apresentaram maiores chances (OR, 3,15; IC 95\%, 2,49-3,99) de desenvolver } \\
\text { TEV. Os procedimentos mais curtos (Percentil <20) foram associados a uma menor } \\
\text { incidência de TEV (OR 0,51; IC 95\% 0,27-0,76) em comparação com aqueles de } \\
\text { duração moderada. As mesmas associações estavam presentes nos modelos ajustados } \\
\text { ao escore de propensão e nos modelos estratificados por subgrupos } \\
\text { de procedimentos craniano, espinhal, nervo periférico e carótida. }\end{array}$ \\
\hline $\begin{array}{l}\text { Artigo 8: } \\
\text { Hamilton, } \\
\text { Hull, 2011 }\end{array}$ & Yee, & $\begin{array}{l}\text { Oito ECRs foram identificados. Seis ECRs envolvendo } 1.170 \text { pacientes avaliaram baixa } \\
\text { dose de heparina não fracionada (HNF) ou HBPM versus um grupo controle. Cinco dos } \\
\text { seis estudos encontraram uma redução significativa no risco de TEV sintomático e } \\
\text { assintomático com profilaxia com heparina. A relação de risco combinada foi de } 0,58 \\
\text { (intervalo de confiança de } 95 \% \text {, 0,45-0,75). ICH foi mais comum naqueles que } \\
\text { receberam heparina, mas não estatisticamente significante. Para cada } 1000 \text { pacientes } \\
\text { que recebem profilaxia com heparina, serão evitados } 91 \text { eventos de TEV } \\
\text { (aproximadamente } 35 \text { dos quais são trombose venosa profunda proximal ou embolia } \\
\text { pulmonar e } 9 \text { a } 18 \text { são sintomáticos), enquanto } 7 \text { ICHs e } 28 \text { sangramentos menores } \\
\text { ocorreram. }\end{array}$ \\
\hline
\end{tabular}




\section{DISCUSSÃO}

No artigo 1 como mostra o quadro, os pacientes neurocirúrgicos e neurocríticos, que estavam com tratamento antiagregante prévio, tinham alguma comorbidade, com aumento do risco de um evento trombótico, de acordo com a literatura. ${ }^{5}$ Observa-se no estudo que quanto maior o número dos fatores de risco (>= a 3 FR), o número de suspensão de antiagregantes antes do procedimento cirúrgico diminuía, considerando também o procedimento cirúrgico. E se o paciente possuía Stent e antiagregante único ou duplo, o retorno das medicações após o procedimento cirúrgico, ocorria frequentemente após $24 \mathrm{~h}$ da cirurgia. ${ }^{(5,6,13)}$

$\mathrm{O}$ artigo 3 revela que dos 11.346 pacientes do estudo, $70(0,6 \%)$ tiveram documentação de doença trombótica, sendo que 28 apresentaram TVP e 42 Embolia Pulmonar (EP). Identifica que pacientes com longa permanência de internação, raça branca, IMC > 30 e idade > 60 anos, tinham um risco maior para TEV. As comorbidades diabetes, câncer ativo e idade superior a 60 anos foram as mais prevalentes. ${ }^{(9)}$

Em relação a uso de tratamento mecânico para os pacientes neurocirúrgicos, o estudo revelou que $80 \%$, dos hospitais mantinham esse método no pré e no pós-operatório. O artigo 6 traz dois protocolos utilizados em momentos diferentes em um departamento de neurocirurgia na França, onde se tem como método primário, associado a métodos farmacológicos, o tratamento mecânico; sendo que no protocolo A havia métodos mecânicos e no protocolo B acrescentou-se a CPI. ${ }^{(5,10,13)}$

No artigo 8, as recomendações da $9^{\text {a }}$ edição da ACCP, demonstram que a CPI deve ser utilizada com ou sem meias compressivas, sendo um ótimo método para prevenção de TVP, com baixo risco de sangramento em pacientes neurocirúrgicos. ${ }^{11}$ Já no artigo 4, há uma forte recomendação para pacientes submetidos a craniotomias eletivas, conforme protocolos citados no estudo, de uso da CPI com HPBM ou HNF 24 horas após o procedimento cirúrgico $^{(7)}$ 


\begin{tabular}{|l|l|}
\hline Protocolo $A$ & Protocolo B \\
\hline $\begin{array}{l}\text { O protocolo consistiu em medidas de profilaxia } \\
\text { mecânicos e farmacológicos constituídos por ES } \\
\text { e, dependendo da estratificação do risco } \\
\begin{array}{l}\text { individual de TV, a administração de HBPM } \\
\text { (Enoxaparina de sódio } 40 \mathrm{mg} / 0,4 \mathrm{ml}) .\end{array}\end{array}$ & $\begin{array}{l}\text { O protocolo consistiu mesmas medidas descritas no } \\
\text { A, além da introdução de dispositivos de CPI em } \\
\text { MMII aplicada no período perioperatório em todos } \\
\text { os doentes, e apenas no grupo de alto risco também } \\
\text { no pós-operatório. }\end{array}$ \\
\hline
\end{tabular}

Fonte: Chibbaro, et al, $2017^{(10)}$

Em relação à farmacologia trombofilática das neurocirurgias, o artigo 2 evidencia que a falta de um antídoto específico para reversão imediata dos efeitos de HBPM, antiplaquetários ou novos anticoagulantes orais, é um dilema em pacientes neurocirúrgicos. A associação de métodos farmacológicos e mecânicos elevam a efetividade da tromboprofilaxia no paciente neurocirúrgico, e em pacientes com elevado risco de trombose é indicada a combinação das meias de compressão graduada com ou sem dispositivo de compressão intermitente, HBPM ou HNF no pós-operatório. ${ }^{(6,13)}$

O estudo traz que em alternativa à anticoagulação dupla (Aspirina e Clopidogrel) em pacientes com Stent recente em artéria coronária, o clopidrogrel pode ser interrompido 5 dias antes de craniotomias e a aspirina deve ser continuada em pacientes com alto risco para sangramento, pois, não só este estudo como todos os demais referem que o risco de sangramento deve ser avaliado individualmente, caso a caso, principalmente em relação a pacientes cardíacos, levando sempre em consideração o quesito risco-benefício. ${ }^{(5,6,13)}$

Diretrizes mostram que de 1000 pacientes que recebem profilaxia de heparina, 91 eventos de TEV serão evitados, e que o risco estimado de TEV com craniotomia para doença não maligna foi relatado em $5 \%$ e para doença maligna em $10 \%$.

No artigo 4, a craniotomia para doença não maligna teve uma das maiores taxas de TEV (6,5\%), no entanto, a incidência de TEV após craniotomia para tumor foi uma das mais baixas. A maior incidência de TEV foi relacionda à cirurgia de deformidade da coluna vertebral $(6,7 \%){ }^{(9,12,14)}$ 
Em relação aos novos anticoagulantes, o artigo 5 revela que no período de 2014, o uso de novos anticoagulantes era reduzido, mas em 2016 houve crescimento significativo da prescrição, e além disso o estudo descreve bem as complicações pós-operatórias de sangramento: de 30 pacientes, 13,3 \% tiveram complicações hemorrágicas, sendo que 2 morreram. A recomendação é que se descontinue a medicação 24 a 48 horas antes do procedimento cirúrgico, pois, o estudo revelou que pacientes com interrupção inferior a 24 horas, as taxas de sangramento aumentaram significativamente. ${ }^{(7-8)}$

Em pacientes anticoagulados submetidos a cirurgias decorrentes de trauma cerebral, a suspensão da anticoagulação antes da cirurgia não ocorre no periodo 24 - 48 horas antes do procedimento, e como não está disponível um reversor de anticoagulação de pronto uso, as hemorragias e complicações mais graves ocorrem neste grupo de pacientes. O retorno da anticoagulação não influenciou em eventos hemorrágicos. Novos anticoagulantes orais (NOACS) foram reiniciados no intervalo de 3-7 dias, tendo menores complicações.

Um estudo na base de dados do NSQIP, de 2005 a 2012, revela que cirurgias prolongadas levam à estresse oxidativo, estase sanguínea, lesão endotelial, associado à imobilização no leito no pós-operatório, com aumento dos eventos trombóticos. Um dado mostra que, de 500.000 hospitalizações, 100.000 mortes por ano ocorrem por tromboembolismo venoso. Esse dado é importante para quantificar gastos, e tomadas de decisões das equipes de saúde, frente a um problema que é quase quem em $100 \%$ é evitável com medidas tromboprofiláticas.

O artigo 3 evidencia que no NSQIP, $1 \%$ dos pacientes desenvolveram um TEV no prazo de 30 dias de pós-operatório. E em outro estudo referido no mesmo artigo, 3,5\% de TEV para aqueles submetidos à craniotomia. ${ }^{(9,14,16-18)}$ 
O NSQIP é um método muito importante para avaliação do risco cirúrgico, no entanto ainda não foi validado no Brasil, sendo considerado ótimo programa a ser utilizado a fim de trazer mais segurança cirúrgica, na avalição perioperatória.

Na maioria dos estudos, os métodos tromboprofiláticos são associados, seja o uso de meias compressivas, CPI, HBPM, HNF ou NOACS, no entanto, os métodos farmacológicos ainda não são totalmente seguros para o uso, pois, como enfatizado nos discursos dos estudos aqui mostrados, os casos de profilaxia farmacológica precisam ser avaliados individualmente. No entanto, se os NOACS, tivessem reversores de pronto uso, e seu custo fosse mais baixo, os pacientes neurocirúrgicos poderiam se beneficiar de seu uso, pois, conforme traz a literatura, sua utilização vem se tornado cada vez maior, e são os medicamentos de escolha para profilaxia em muitos procedimentos cirúrgicos. ${ }^{(5-7,10-13)}$

Há tanto que se levar em consideração do paciente neurocirúrgico que o American College of Chest Physicians (ACCP), elaborou uma Diretriz amplamente utilizada no mundo. Atualmente a nona edição da “Terapia Antitrombótica e Prevenção de Trombose: Diretrizes de Prática Clínica com Base em Evidências do ACCP (ACCP), ainda é bastante utilizada. O estudo de Gould et al, 2012(15) aponta as recomendações expostas no Quadro 2 de forma resumida. 
Quadro 2: Recomendações de terapia antitrombótica segundo Gould et al, 2012 ${ }^{(15)}$

\begin{tabular}{|c|c|}
\hline Procedimentos Neurocirúrgicos & Recomendações (Grau 2C) \\
\hline Pacientes submetidos à Craniotomia & $\begin{array}{l}\text { Profilaxia mecânica, preferencialmente com CPI, com } \\
\text { ou sem profilaxia farmacológica }\end{array}$ \\
\hline $\begin{array}{l}\text { Craniotomia com risco alto para TEV (exemplo } \\
\text { daqueles submetidos à craniotomia para doença } \\
\text { maligna) }\end{array}$ & Profilaxia farmacológica e profilaxia mecânica \\
\hline Cirurgia espinhal contínua & $\begin{array}{l}\text { Profilaxia mecânica, preferível ao CPI, sem profilaxia, } \\
\text { sem nenhuma profilaxia farmacológica. HNF ou HBPM }\end{array}$ \\
\hline $\begin{array}{l}\text { Cirurgia espinhal com alto risco para TEV (Incluindo } \\
\text { aqueles com doença maligna ou aqueles submetidos a } \\
\text { cirurgia com abordagem anterior-posterior combinada) }\end{array}$ & Profilaxia farmacológica à profilaxia mecânica \\
\hline $\begin{array}{l}\text { Pacientes com trauma grave: Lesão traumática cerebral, } \\
\text { Lesão espinhal aguda, e Lesão traumática de coluna: }\end{array}$ & $\begin{array}{l}\text { HNF em baixas doses, HBPM ou profilaxia mecânica, } \\
\text { preferencialmente com CPI, sem outra profilaxia }\end{array}$ \\
\hline $\begin{array}{l}\text { Pacientes de trauma grave de alto risco para TEV } \\
\text { (incluindo aqueles com lesão aguda de medula espinhal, } \\
\text { lesão cerebral traumática e trauma de cirurgia espinhal) }\end{array}$ & $\begin{array}{l}\text { Adicionar profilaxia mecânica à profilaxia } \\
\text { farmacológica quando não contraindicada por lesão de } \\
\text { extremidade inferior }\end{array}$ \\
\hline $\begin{array}{l}\text { Para os principais pacientes de trauma em que HBPM e } \\
\text { LDUH são contraindicados: }\end{array}$ & $\begin{array}{l}\text { Profilaxia mecânica, preferencialmente com CPI, sem } \\
\text { profilaxia, quando não contraindicado por lesão de } \\
\text { extremidade inferior. Sugere-se adicionar profilaxia } \\
\text { farmacológica com HBPM ou HNF em baixas doses, } \\
\text { quando o risco de sangramento diminui ou a } \\
\text { contraindicação a heparina é resolvida. }\end{array}$ \\
\hline Para os principais pacientes com trauma & $\begin{array}{l}\text { Filtro de Veia Cava Inferior não deve ser usado para a } \\
\text { prevenção primária de TEV. }\end{array}$ \\
\hline Para pacientes com trauma maior & $\begin{array}{l}\text { Vigilância periódica com ultrassom de compressão } \\
\text { venosa não deve ser realizada }\end{array}$ \\
\hline Pacientes submetidos à craniotomia & $\begin{array}{l}\text { Profilaxia mecânica, preferencialmente com CPI, com } \\
\text { ou sem profilaxia farmacológica }\end{array}$ \\
\hline $\begin{array}{l}\text { Craniotomia com risco alto para TEV (exemplo } \\
\text { daqueles submetidos à craniotomia para doença } \\
\text { maligna) }\end{array}$ & Profilaxia farmacológica e profilaxia mecânica \\
\hline Cirurgia espinhal contínua & $\begin{array}{l}\text { Profilaxia mecânica, preferível ao CPI, sem profilaxia, } \\
\text { sem nenhuma profilaxia farmacológica. HNF ou HBPM }\end{array}$ \\
\hline $\begin{array}{l}\text { Cirurgia espinhal com alto risco para TEV (Incluindo } \\
\text { aqueles com doença maligna ou aqueles submetidos a } \\
\text { cirurgia com abordagem anterior-posterior combinada) }\end{array}$ & Profilaxia farmacológica à profilaxia mecânica \\
\hline $\begin{array}{l}\text { Pacientes com trauma grave: Lesão traumática cerebral, } \\
\text { Lesão espinhal aguda, e Lesão traumática de coluna: }\end{array}$ & $\begin{array}{l}\text { HNF em baixas doses, HBPM ou profilaxia mecânica, } \\
\text { preferencialmente com CPI, sem outra profilaxia }\end{array}$ \\
\hline $\begin{array}{l}\text { Pacientes de trauma grave de alto risco para TEV } \\
\text { (incluindo aqueles com lesão aguda de medula espinhal, } \\
\text { lesão cerebral traumática e trauma de cirurgia espinhal) }\end{array}$ & $\begin{array}{l}\text { Adicionar profilaxia mecânica à profilaxia } \\
\text { farmacológica quando não contraindicada por lesão de } \\
\text { extremidade inferior }\end{array}$ \\
\hline $\begin{array}{l}\text { Para os principais pacientes de trauma em que HBPM e } \\
\text { LDUH são contraindicados: }\end{array}$ & $\begin{array}{l}\text { Profilaxia mecânica, preferencialmente com CPI, sem } \\
\text { profilaxia, quando não contraindicado por lesão de } \\
\text { extremidade inferior. Sugere-se adicionar profilaxia } \\
\text { farmacológica com HBPM ou HNF em baixas doses, } \\
\text { quando o risco de sangramento diminui ou a } \\
\text { contraindicação a heparina é resolvida. }\end{array}$ \\
\hline Para os principais pacientes com trauma & $\begin{array}{l}\text { Filtro de Veia Cava Inferior não deve ser usado para a } \\
\text { prevenção primária de TEV. }\end{array}$ \\
\hline Para pacientes com trauma maior & $\begin{array}{l}\text { Vigilância periódica com ultrassom de compressão } \\
\text { venosa não deve ser realizada }\end{array}$ \\
\hline
\end{tabular}




\section{CONCLUSÃO}

Os estudos buscam tromboprofilaxia ideal e a garantia da hemostasia dos pacientes neurocirúrgicos, tentando sempre balancear o quesito risco-benefícios de todos os métodos. Fica claro que cada paciente e cada procedimento neurocirúrgico tem de ser avaliado de forma individualizada, não levando em consideração apenas o procedimento em si, como também as avaliações laboratoriais do paciente que será submetido ao procedimento.

O uso de CPI tem sido um método de escolha primária na tromboprofilaxia dos pacientes neurocirúrgicos, sendo aplicada de forma isolada, com meias de compressão graduada ou associada aos métodos farmacológicos. Este estudo conclui que os NOACS precisam de mais estudos para serem usados de forma segura nos pacientes neurocirúrgicos, pois o tempo adequado de suspensão antes procedimento cirúrgico não ficou claro, e por isso o ideal seria ampliar investigações acerca da matéria, considerando que trata-se de medicação utilizada rotineiramente, nos procedimentos cirúrgicos em geral.

Mais investigações são necessárias para avaliar qual o método mais seguro para se utilizar em neurocirurgia, visto que em alguns estudos, o TVP e a TEV ainda são considerados diagnósticos não tratados com o cuidado necessário, apesar do número crescente de óbitos e hospitalizações. E com isso é necessário que a avaliação perioperatória dos pacientes, neurocirúrgicos ou não, seja realizada de acordo com as Diretrizes de Tromboprofilaxia, para os pacientes cirúrgicos.

\section{REFERÊNCIAS}

1. Goldman, L. Schafer, A.I., MD. Cecil Medicina. 24º ed. Rio de Janeiro: Elsevier; 2014.

2. Gomes M, Ramacciotti E. Programa de Auto-Avaliacão em Cirurgia Tromboembolismo venoso. Rio de Janeiro: Diagrafiq. 2012; 185p. 
3. Rassam E, Pinheiro TC, Stefan LFB, Módena SF. Complicações tromboembólicas no paciente cirúrgico e sua profilaxia. ABCD Arq Bras Cir Dig 2009;22(1):41-4.

4. Hill J, Treasure T. Reducing the risk of venous thromboembolism (deep vein thrombosis and pulmonary embolism) in patients admitted to hospital: summary of the NICE guideline. Heart. 2010; 96(11):879-82.

5. Alonso, EV, Fábregas N, Maceiras PR, et al. National survey on thromboprophylaxis and anticoagulant or antiplatelet management in neurosurgical and neurocritical patients. Rev Esp Anestesiol Reanim. 2015; 62(10):557-64.

6. Niemi T, Armstrong E. Thromboprophylactic management in the neurosurgical patient with high risk for both thrombosis and intracranial bleeding. Current Opinion in Anaesthesiology. 2010; 23(5):558-63.

7. Robba C, Bertuetti R, Rasulo F, Bertuccio A, Matta E B. Coagulation management in patients undergoing neurosurgical procedures. Curr Opin Anaesthesiol. 2017; 30(5):527-33.

8. Croci DM, Kamenova M, Guzman R, Mariani L, Soleman J. Novel Oral Anticoagulants in Patients Undergoing Cranial Surgery. Neurocirurgião Mundial. 2017; 105:841-8.

9. Agarwal N, Zenonos GA, Agarwal P, Walch FJ, Roach E, Stokes SJ, et al. Risk-to-Benefit Ratio of Venous Thromboembolism Prophylaxis for Neurosurgical Procedures at a Quaternary Referral Center. Neurosurgery, 2019; 84(2):355-61.

10. Chibbaro S, Cebula H, Todeschi J, Fricia M, Vigouroux D, Abid H, et al. Evolution of Prophylaxis Protocols for Venous Thromboembolism in Neurosurgery: Results from a Prospective Comparative Study on Low-Molecular-Weight Heparin, Elastic Stockings, and Intermittent Pneumatic Compression Devices. World Neurosurg. 2018; 109:e510-e516.

11. Hamilton MG, Yee WH, Hull RD. Venous Thromboembolism Prophylaxis in Patients Undergoing Cranial Neurosurgery: A Systematic Review and Meta-analysis. Neurosurgery. 2011; 68(3):571-81. 
12. Bekelis K, Labropoulos N, Coy S. Risk of Venous Thromboembolism and Operative Duration in Patients Undergoing Neurosurgical Procedures. Neurosurgery, 2017; 80 (5):787-92

13. Khan NR, Patel PG, Sharpe JP, Lee SL, Sorenson J. Chemical venous thromboembolism prophylaxis in neurosurgical patients: an updated systematic review and meta-analysis. Journal of Neurosurgery, 2018; 129(4):906-15.

14. Nyquist P, Bautista C, Jichici D, Burns J, Chhangani S, DeFilippis M, et al. Prophylaxis of venous thrombosis in neurocritical care patients: an executive summary of evidence-based guidelines: a statement for health professionals from the Society of Neurocritical Care and Society of Critical Care Medicine. Neurocrit Care. 2017; Crit Care Med. 2017; 45(3):476-9.

15. Gould MK, Garcia DA, Wren SM, et al. Prevention of VTE in nonorthopediic surgical patients: antithrombotic therapy and prevention of thrombosis, $9^{\circ}$ ed: American College of Chest Physicians Evidence-Based Clinical Practice Guidelines. Peito. 2012; 141(2 Suppl): e227Se277S.

16. Guyatt GH, Akl EA, Crowther M, Gutterman DD, Schuünemann HJ. Executive summary: Antithrombotic Therapy and Prevention of Thrombosis, 9th ed: American College of Chest Physicians Evidence-Based Clinical Practice Guidelines. Chest. 2012 Feb;141(2 Suppl):7S$47 \mathrm{~S}$.

17. Rinaldo L, Brown DA, Bhargav AG, Rusheen AE, Naylor RM, Gilder HE, et al. Venous thromboembolic events in patients undergoing craniotomy for tumor resection: incidence, predictors, and review of literature. J Neurosurg. 2019; 132(1):10-21.

18. Farr S, Toor H, Patchana T, Podkovik S, Wiginton JG, Sweiss R, et al. Risks, Benefits, and the Optimal Time to Resume Deep Vein Thrombosis Prophylaxis in Patients with Intracranial Hemorrhage. Cureus. 2019;11(10):e5827. 